\title{
Assessment of serum concentrations of matrix metalloproteinase 1 , matrix metalloproteinase 2 and tissue inhibitors of metalloproteinases 1 in atopic dermatitis in correlation with disease severity and epidermal barrier parameters
}

\author{
Magdalena Basałygo', Joanna Śliwińska', Magdalena Żbikowska-Gotz², Kinga Lis², Ewa Socha², Zbigniew Bartuzi², \\ Barbara Zegarska ${ }^{1}$
}

'Department of Cosmetology and Aesthetic Dermatology, Ludwik Rydygier Collegium Medicum in Bydgoszcz, Nicolaus Copernicus University in Torun, Poland

${ }^{2}$ Department and Clinic of Allergology, Clinical Immunology and Internal Diseases, Ludwik Rydygier Collegium Medicum in Bydgoszcz, Nicolaus Copernicus University in Torun, Poland

Adv Dermatol Allergol 2021; XXXVIII (5): 773-779

DOI: https://doi.org/10.5114/ada.2021.110043

\begin{abstract}
Introduction: Matrix metalloproteinases (MMPs) are a group of proteolytic enzymes, conditioning the integrity of skin cells, however, their role in the inflammatory process of atopic dermatitis (AD) and the direct effect on the epidermal barrier parameters remain unexplained.

Aim: To assess MMP-1, MMP-2, tissue inhibitors of metalloproteinases (TIMP)-1 concentrations in blood serum in the context of transepidermal water loss (TEWL) and stratum corneum hydration in AD. Moreover, serum levels of MMPs and TIMP-1 were analysed in relation to the Eczema Area and Severity Index (EASI).

Material and methods: Forty-three AD patients and 22 control group subjects have been investigated. Serum concentrations of MMP-1, MMP-2, and TIMP-1 have been evaluated with ELISA. TEWL and stratum corneum hydration have been assessed with a TM300 Tewameter and a CM825 Corneometer. Skin lesions in patients with AD have been evaluated with the Eczema Area and Severity Index.

Results: MMP-1 and MMP-2 serum concentrations were significantly higher in the AD group. The results of TIMP-1 serum concentration were similar for both groups. The correlation between the serum concentration and the EASI was demonstrated only for MMP-2 for patients with severe and moderate AD. Patients with AD and TIMP-1 serum concentration greater than MMP-1 presented lower TEWL and higher epidermal hydration.

Conclusions: The results of this study warrant further investigation. The predominance of TIMP-1 over MMP-1 in blood serum can potentially limit TEWL and maintain the proper water content of the epidermis. Future work is necessary to establish how reliable the role of MMP-2 concentration is as an indicator of the severity of AD.
\end{abstract}

Key words: atopic dermatitis, matrix metalloproteinases 1, matrix metalloproteinases 2, tissue inhibitors of metalloproteinase 1, transepidermal water loss.

\section{Introduction}

Atopic dermatitis (AD) is a chronic and recurrent inflammatory dermatosis affecting $2.0-17.6 \%$ of adults, depending on the region and method of assessment [1]. The pathogenesis of the disease stems from genetic and environmental factors, immune dysregulation and epi- dermal barrier disorders, which are currently the subject of many studies. Heterogeneous symptoms include erythema, pruritus, dry skin, lichenification lesions - plaques or prurigo nodules, which are characterized by fibrosis in the dermis [2]. Long-term epidermal barrier defects, chronic skin exposure to allergens, itching and scratch-

Address for correspondence: Magdalena Basałygo, Department of Cosmetology and Aesthetic Dermatology, Ludwik Rydygier Collegium Medicum, Nicolaus Copernicus University, 13-15 Jagiellońska St, 85-067 Bydgoszcz, Poland, e-mail: magdalena_basalygo@poczta.onet.pl Received: 16.03.2020, accepted: 25.04.2020. 
ing, frequently alongside coexisting bacterial infections play a contributing role [3, 4]. Factors disturbing the microbiological balance (including S. aureus) appear before the onset of $A D$ symptoms and lead to an increase in transepidermal water loss (TEWL) [5]. Reports in the literature indicate that lichenification changes are a consequence of changes in the matrix metalloproteinases (MMPs)/tissue inhibitors of metalloproteinase (TIMP) concentration balance $[6,7]$.

The extracellular matrix (ECM) is an active structure containing lipids, enzymes, structural proteins and antimicrobial peptides [8]. All its components are necessary to maintain the correct barrier function, especially important during the course of AD [9]. Extracellular matrix metalloproteinases and their inhibitors are responsible for the reconstruction of matrix components and their remodelling [10]. Matrix degradation by MMPs leads to damage to the corneodesmosomes, which in turn can cause impaired water retention [11]. ECM degradation also leads to loss of ligand function for integrins [12], which are present in the base level of the epidermal cells, which in turn may be important in the process of wound healing in $A D$ [13]. Integrins enable the interaction between keratinocytes and the extracellular matrix, participate in the formation of adhesive connections, desmosomes, hemidesmosomes and take part in the immune response to the inflammatory process by attaching the leukocyte to the endothelium [14].

The participation of MMPs in the immune regulation of the inflammatory process enables leukocyte movement to the site of the inflammatory reaction [15]. MMP-1 from the collagenase subgroup $[15,16]$ and MMP-2 from the gelatinase subgroup [17] are significant from the AD etiopathogenesis' perspective. MMP-1 is a proteolytic enzyme containing a hemopexin-like domain, which is necessary for the degradation of the collagen triple helix. The unwinding of the collagen triple helix leads to an increased susceptibility of collagen to degradation by other proteases, e.g. by MMP-2 action [18]. MMP-2 on the other hand cleaves type II and III collagen [19]. The activity of extracellular matrix metalloproteinases is regulated through interaction with their natural inhibitors e.g. TIMP-1, reversible binding in a $1: 1$ molar ratio [20]. In physiological processes, the presence of MMPs leads to anti-inflammatory and protective effects [21], while changes in the expression or activity of MMPs/TIMP contribute to an imbalance between degradation processes and the synthesis of extracellular matrix components, which result in pathological processes in tissues [22].

A rising number of scientific reports postulate that maintaining the MMPS/TIMP balance enables the proper function of the epidermal barrier [22, 23]. Proteases are involved in the exfoliation process [22] and require the correct stratum hydration gradient to be maintained [23]. Current knowledge recognises their participation in the course of diseases such as psoriasis, rosacea, bullous dis- eases, scleroderma and leg ulcers [24], however, little is known about their role in AD.

\section{Aim}

The core aim of the study was determination and comparison of the concentrations of MMP-1, MMP-2 and TIMP-1 in blood serum in people with confirmed diagnosis of $A D$ and in healthy population. A secondary aim was to investigate the interdependencies between MMP-1, MMP-2 and TIMP-1 levels and Eczema Area and Severity Index (EASI) disease activity index. Moreover, the study examined whether the postulated concentration interdependencies may have an effect on epidermal barrier parameters assessed by TEWL measurement and the skin hydration test.

\section{Material and methods}

The study group consisted of 43 patients with $A D$ diagnosis, aged between 18 and 35 (average age of 26). The patient population consisted of 20 females and 23 males (47\% and 53\%, respectively). The control group comprised 22 healthy volunteers, declaring no personal or family history of dermatological diseases. It consisted of 12 females and 10 males aged 22 to 38 (average age of 29) selected to match the age and gender profile of the patient group. The research was conducted from January to June 2016. Diagnosis of atopic dermatitis was based on Hanifin and Rajka criteria. Food allergies were diagnosed in $28 \%$ of cases and the sensitization to inhalant allergens was recorded in 36\% of the patients with AD. Furthermore, 19\% suffered from bronchial asthma and rhinitis was observed in $44 \%$ (specifically, non-allergic rhinitis in $27 \%$ and allergic rhinitis in $17 \%$ of the group). Recorded answers concerning the possible hypersensitivity reactions were taken as reflecting patient's history and were not based on results of diagnostic procedures such as specific IgE, skin prick tests or challenge tests. The inclusion criteria for the study for both groups were: participant's age (over 18 years) and provision of written consent to participate in the study. In addition, enrolled patients could not have undergone any general treatment for at least 1 month before the participation in the study. Furthermore the study excluded patients with $A D$ undergoing phototherapy, treatment with cyclosporine A, oral and topical corticosteroids (and for up to 6 weeks after stopping the therapy), topical calcineurin inhibitors such as pimecrolimus and tacrolimus, patients undergoing or who had previously completed, a specific immunotherapy, breastfeeding and pregnant women and patients with inflammatory, infectious, autoimmune and cancer diseases. The exclusion criteria extended to the use of emollients on the day of the study as well as birthmarks, scars, and injuries in the examined area.

Evaluation of serum MMP-1, MMP-2 and TIMP-1 concentrations by immunoenzymatic method (ELISA) was 
carried out using commercial Human Platinum ELISA kits (from eBioscience). Sera were aliquoted into Eppendorf tubes and preserved frozen at $-82^{\circ} \mathrm{C}$ until determinations were made. The measurements were carried out in accordance with the guidelines given by the kit manufacturer.

Assessment of the severity of skin lesions in patients with AD was based on the EASI. The EASI test consists of assessing the severity of the inflammation of the skin divided into four areas: head/neck, torso, upper and lower limbs. Each of the above-mentioned areas was subjected to a fourstage evaluation (awarding 0-3 points), taking into account each of the four types of eruptions: erythema, infiltration/ papules, erosions/excoriations and lichenification. The evaluated efflorescence was correlated with the affected body surface in each area assessed, awarding 0-6 points. The maximum result measured by the EASI was 72 points.

Measurement of the degree of damage to the epidermal barrier in the area of skin affected by $A D, 2 \mathrm{~cm}$ from the inflammatory focus and on the same area of a seemingly healthy skin was carried out for the determination of TEWL, using the TM 300 Tewameter connected to the Cutage MPA 58 adapter from Courage-Khazaka (Cologne, Germany). All measurements were made at room temperature, i.e. $20-22^{\circ} \mathrm{C}$, and $40-60 \%$ relative humidity, continuously monitored by the RHT 100 ambient conditions sensor. To ensure stability of measurements, the probe was kept at a temperature closely matching the skin temperature using a Probe Heater PR 100. The study was conducted on a non-dominant upper limb, on the area of skin with no hair. In order to further ensure the accuracy of the readings, all parameters were measured at the same time of a day and in consistent lighting conditions, and the test subjects were allowed to rest in the examination room for 20 min before the tests began. Each examined area was subjected to 25 consecutive measurements at 1 s intervals, taking their average value as the calculated result. The values of TEWL level measurements are presented in units of gram per square meter per hour $\left[\mathrm{g} / \mathrm{m}^{2} / \mathrm{h}\right]$, consistently with the test equipment manufacturer's guidelines.

The measurement of epidermal hydration was carried out using a Corneometer CM 825. The apparatus headpiece was placed at a right angle to the examined skin area, with a force adjusted by the spring tension in the probe. The final score of the degree of hydration of the epidermis was an average of three consecutive 1-second measurements carried out on each tested area. Each individual measurement was taken from a distance of $2 \mathrm{~mm}$ above the skin, to minimize the risk of occlusion developing under the head of the probe. The results of the corneometric measurements were presented in arbitrary units in the range of 0-130 a.u.

\section{Statistical analysis}

The obtained data for the TEWL measurements and the measurements of skin hydration were recorded in MPA software and subjected to statistical analysis us- ing PQStat version 1.6.2.901 and Microsoft Excel. The two studied groups were compared using the non-parametric U-Man-Whitney test. Interdependencies between parameters were examined based on either Spearman Rank or Pearson correlation coefficients. Data with a statistical significance level below $0.05(p<0.05)$ were considered statistically significant, while data with a statistical significance level below $0.01(p<0.01)$ were considered highly significant.

\section{Results}

The average clinical condition of patients according to the EASI index was 17.96 points with standard deviation \pm 15.36 ; with a minimum value of 0.8 and a maximum of 66.8 points, and the median of 24.2 points. In the group of patients with $A D$, the average TEWL value (with standard deviation) within the affected skin was $41.5 \pm 15.7 \mathrm{~g} / \mathrm{m}^{2} / \mathrm{h}$; at $2 \mathrm{~cm}$ away from the lesion: $37.07 \pm 15.15 \mathrm{~g} / \mathrm{m}^{2} / \mathrm{h}$; and within the seemingly healthy skin: $18.91 \pm 6.9 \mathrm{~g} / \mathrm{m}^{2} / \mathrm{h}$. The control group had an average TEWL value of $13.7 \pm 5.12 \mathrm{~g} / \mathrm{m}^{2} / \mathrm{h}$, and all results in this group were within the normal range $\left(<25 \mathrm{~g} / \mathrm{m}^{2} / \mathrm{h}\right)$. The average value of the measurement of skin hydration in the examined group, was found to be $18.76 \pm 7.06$ a.u. for the affected skin; $24.28 \pm 5.78$ a.u. at $2 \mathrm{~cm}$ away from the lesion; and $29.44 \pm 5.22$ a.u. on the seemingly healthy skin. The level of hydration of the stratum corneum in the control group was $48.57 \pm 5.57$ a.u.

Statistically significant differences were observed between $A D$ patients and healthy subjects only in the case of MMP-1 (Table 1) and MMP-2 (Table 2). Patients with atopic dermatitis showed significantly increased levels of MMP-1 $(Z=2.32, p=0.02)$ and MMP-2 $(Z=1.4, p=0.04)$ in serum, as compared to the control group. Changes in MMP-1 concentration in relation to the severity of skin lesions (EASI Index) showed no correlation (Figure 1). For

Table 1. Non-parametric analysis, using Mann-Whitney $U$ test, of plasma MMP-1 values, in peripheral blood of patients with $A D$ vs. control subjects

\begin{tabular}{llcc}
\hline Variables & & \multicolumn{2}{c}{ Patients } \\
\cline { 2 - 4 } & & \multicolumn{1}{c}{$\begin{array}{c}\text { Atopic } \\
\text { dermatitis }\end{array}$} & $\begin{array}{c}\text { Control } \\
\text { group }\end{array}$ \\
\hline MMP-1 & $N$ & 43 & 22 \\
\cline { 2 - 4 } & Min. & 394.37 & 64.02 \\
\cline { 2 - 4 } & Max. & 3278.72 & 2767.69 \\
\cline { 2 - 4 } & Median & 117.93 & 698.56 \\
\cline { 2 - 4 } & Mean & 1278.77 & 911.51 \\
\cline { 2 - 4 } & SD & 666.71 & 662.7 \\
\hline Mann-Whitney & Z & - & 2.32 \\
\cline { 2 - 4 } & P-value & - & 0.02 \\
\hline
\end{tabular}

$\overline{n-\text { number of respondents, SD - standard deviation, } Z \text { - Mann-Whitney }}$ $U$ test, $p$ - significance level. 
Table 2. Non-parametric analysis, using Mann-Whitney $U$ test, of plasma MMP-2 values, in peripheral blood of patients with $A D$ vs. control subjects

\begin{tabular}{|c|c|c|c|}
\hline \multirow[t]{2}{*}{ Variables } & & \multicolumn{2}{|c|}{ Patients } \\
\hline & & $\begin{array}{c}\text { Atopic } \\
\text { dermatitis }\end{array}$ & $\begin{array}{c}\text { Control } \\
\text { group }\end{array}$ \\
\hline \multirow[t]{6}{*}{ MMP-2 } & $N$ & 43 & 22 \\
\hline & Min. & 3.5 & 3.5 \\
\hline & Max. & 20472.99 & 796.35 \\
\hline & Median & 3.5 & 3.5 \\
\hline & Mean & 1312.58 & 85.09 \\
\hline & SD & 3901.15 & 202.78 \\
\hline \multirow{2}{*}{$\begin{array}{l}\text { Mann-Whitney } \\
U \text { test }\end{array}$} & $Z$ & - & 1.42 \\
\hline & $P$-value & - & 0.039 \\
\hline
\end{tabular}

$n$ - number of respondents, SD - standard deviation, $Z$ - Mann-Whitney $U$ test, $p$ - significance level.

MMP-2, a negative correlation was obtained with respect to the severity of clinical disease symptoms, but without statistical significance $(r=-0.2, p>0.05)$. An increase in average MMP-2 concentration was more pronounced for patients with EASI $>25$ points, indicating that a correlation with clinical disease activity was only significant in patients with severe and moderate $\operatorname{AD}(r=-0.3$, $p<0.001)$. Although the TIMP-1 concentration presented in Table 3 was found to be higher in the group of patients with AD compared to the control group, it did not exceed the lower limit of detection for the Mann-Whitney test $(p>0.05)$. Similarly, in the clinical assessment, no statistically significant correlation with the extent of skin lesions was found using the EASI index (Figure 1).

Changes in epidermal barrier parameters were also assessed in relation to the concentrations of MMP-1, MMP-2, and TIMP-1. There was no observed effect of the MMP-1 concentration on TEWL level or on the measure-

Table 3. Serum levels of tissue inhibitors of matrix metalloproteinases (TIMP-1) in patients with atopic dermatitis (AD) and control subjects

\begin{tabular}{|c|c|c|c|}
\hline \multirow[t]{2}{*}{ Variables } & & \multicolumn{2}{|c|}{ Patients } \\
\hline & & Atopic dermatitis & Control group \\
\hline \multirow[t]{6}{*}{ TIMP-1 } & $N$ & 43 & 22 \\
\hline & Min. & 180.44 & 254.63 \\
\hline & Max. & 1232.34 & 952.53 \\
\hline & Median & 487.96 & 435.71 \\
\hline & Mean & 568.88 & 462.19 \\
\hline & SD & 291.08 & 186.64 \\
\hline \multirow{2}{*}{$\begin{array}{l}\text { Mann-Whitney } \\
U \text { test }\end{array}$} & $Z$ & - & 1.1322 \\
\hline & $P$-value & - & 0.2575 \\
\hline
\end{tabular}

$n$ - number of respondents, SD - standard deviation, Z - Mann-Whitney $U$ test, $p$ - significance level.

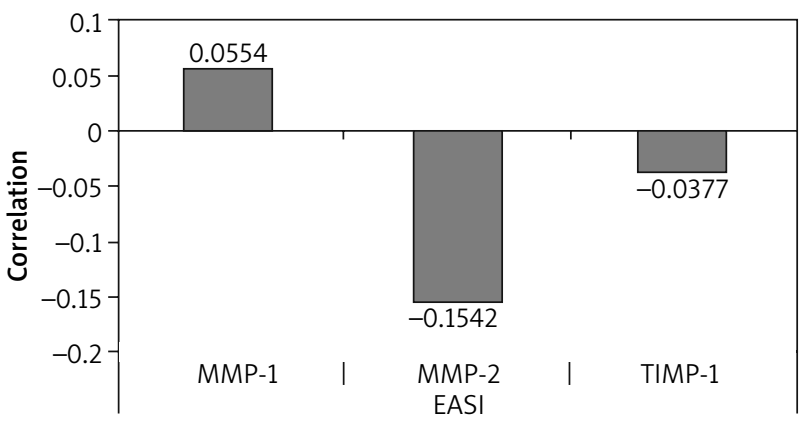

Figure 1. Correlation of serum levels of MMP-1, MMP-2, TIMP-1 and the eczema area and severity index (EASI)

ment of epidermal hydration $(p>0.05)$. On the other hand, a correlation was found between Tewameter measurement results and the epidermis hydration in the case of MMP-2. At the AD-affected area, a strong correlation was found with the TEWL measurement: Spearman's rank correlation coefficient of $0.64(p<0.05)$. An average correlation $(r=0.49, p<0.05)$ was noted at $2 \mathrm{~cm}$ away from the lesions, and no correlations were found $(r=0.1$, $p=0.044)$ only in case of the seemingly healthy skin. On the other hand, a negative correlation was observed in the measurement of epidermal hydration, the Spearman rank correlation coefficient was $r=-0.52, p=0.046$ for AD-affected skin, and $r=-0.39$ for the places $2 \mathrm{~cm}$ away from the lesions. For a seemingly healthy skin, as in case of the TEWL measurement, no correlation was observed.

The ratio of TIMP-1/MMP-1 values was significantly higher in individuals with AD compared to the control group (Mann-Whitney test, $Z=2.36, p=0.014$ ). Further analysis showed that the TEWL level is statistically significantly lower within the affected skin and at a distance of $2 \mathrm{~cm}$ from the lesions when the TIMP concentration is higher than the MMP-1 concentration (Mann-Whitney test, $Z=1.624, p<0.05$ ). The correlations were only $a b$ sent in case of the seemingly healthy skin. The results are presented in Figure 2.

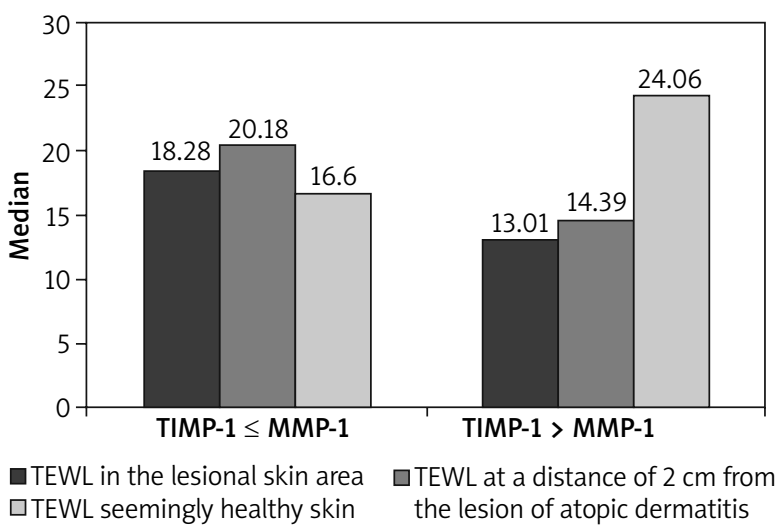

Figure 2. The values (mean \pm SD) of TEWL and comparison with the TIMP-1/MMP-1 ratio patients with $A D$ 
The measurement of epidermal hydration yielded a higher level of hydration within the AD-affected skin and at $2 \mathrm{~cm}$ from the lesions when the TIMP concentration was higher than the MMP-1 concentration (MannWhitney test, $Z=1.936, p<0.05)$. For a seemingly healthy skin, no correlation was found either. The results of the hydration measurements of the epidermis are illustrated in Figure 3.

\section{Discussion}

Atopic dermatitis (AD) is a chronic inflammation of the skin that is characterized by a defective skin barrier [1]. Despite the well-known role of proteases in maintaining the skin barrier, relatively little is known about their involvement in AD. Recent clinical and experimental evidence indicates that MMPs and TIMPs may be the cause of interference with barrier function [25]. Many investigators claim that the activity of proteolytic enzymes may fluctuate in atopic dermatitis and impaired skin barrier function may be the cause of their excessive expression $[9,25]$. Based on these observations, the aim of the current study was to examine the level of MMP-1, MMP-2 and TIMP-1 in blood serum. Hypothesizing that increased MMPs activity may cause excessive exfoliation [11] and impaired skin barrier function may be the cause of their excessive expression [11, 25], a secondary goal was to assess the impact of the above MMPS and TIMP on epidermal barrier parameters in people with AD.

Harper et al. [25] observed an increase in MMP-1 and MMP-3 concentration together with the severity of atopic dermatitis. The severity of the AD process, as demonstrated in another study [26], is associated with the severity of epidermal barrier damage. Atopic skin shows reduced thickness, along with reduced expression of collagen - IV and integrins [8]. This observation could suggest that higher concentrations of MMP-1, MMP-2 and also their inhibitors may correlate with the degree of epidermal barrier damage expressed by TEWL and corneometric measurement.

The present study showed statistically significant elevated concentration levels of MMP-1, MMP-2 notably the level of MMP-2 was several times higher in the examined AD patient group compared to the control group. MMP-2 is often localized in skin lesions associated with atopic dermatitis, but its involvement remains unknown [27, 28]. Studies conducted on a mouse model suggest that MMP-2 may be an indicator of an active inflammatory process in the course of AD $[29,30]$. MMP-2 defect was also observed by Li et al. [28]. Moreover, the ability of the MMP2 inhibitor to restore the integrity of keratinocytes and skin remodelling prompted the authors of the study to examine the barrier function by TEWL measurement [30]. It was shown that the level of trans-epidermal water loss from the skin treated with the MMP-2 inhibitor was significantly reduced. The authors emphasize that in order

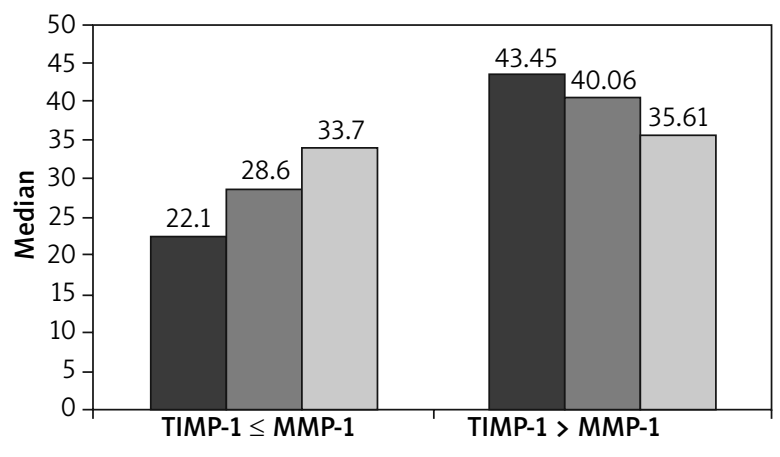

$\square$ Skin hydration level in the lesional skin area

$\square$ Skin hydration level on the seemingly healthy skin

Figure 3. The values of measurement of hydration in the stratum corneum with the Corneometer and comparison with the TIMP-1/MMP-1 ratio patients with $\mathrm{A}$

to understand the molecular genesis of $A D$, the consideration of underlying mechanisms, such as TEWL, may need more attention, and may be targeted by therapy in patients. The suggested interdependency is not entirely consistent with the results of our own research, which confirmed the difference between MMP-2 concentration in serum of patients with AD and healthy population, but no correlation between MMP-2 concentration and epidermal barrier parameters was found. However, a statistically significant correlation was demonstrated between MMP/TIMP and TEWL and epidermal hydration measurements. A lower TEWL value was measured when the TIMP-1 concentration exceeded the MMP-1 concentration. The data suggest that the barrier function of the epidermis is regulated not only by the concentration of MMP-1, but also by TIMP-1. The predominance of TIMP over MMP prevents proteolytic degradation and causes the accumulation of extracellular matrix components [31]. In the studies carried out by Titz et al. [32], there were no differences in the concentration of MMP-2, TIMP-1 between patients with atopic dermatitis and the control group, and no correlation with the severity of the disease was found, which remains in partial agreement with our own research. Instead, there was a positive correlation between MMP-2 and TIMP-1 in the control group. In turn, Katoh et al. [6] found that elevated TIMP-1 levels are characteristic of lichenification and prurigo lesions occurring in chronic AD, so long-term elevated TIMP-1 secretion compared to MMPs may cause basement thickening and skin fibrosis [32]. The authors also add that this may limit the harmful effects of topical glucocorticosteroids consisting of thinning of the epidermis layers, which seems important in the treatment of AD. This may also be confirmed by the use of tacrolimus in the treatment of $A D$, which reverses the regulation of MMP-1/TIMP-1, induced by TGF- $\beta 1$ [33]. After using this drug, no thinning of the epidermis is observed [34], which 
confirms the protective role of balance of MMP-1/TIMP-1 concentration in maintaining the normal epidermal barrier. Additional evidence is found in a study by Mempel et al. [35], where the application of the therapeutic dose of UVA radiation led to an increase in TIMP-1 plasma concentration without change in MMP-1 concentration. Based on observation of ustekinumab treatment, it can be assumed that inhibition of MMPs affects the final phase of keratinocyte differentiation and, as a consequence, tends to reduce the thickness of epidermidis [36].

The body of research presented above proves the importance of MMPS/TIMP balance in the course of $A D$, which is directly related to the maintenance of the normal epidermal barrier. Individual MMP-1 and TIMP-1 concentrations did not differ in the study between the patient and control groups, and were not associated with TEWL and the level of hydration of the epidermis, which could suggest that the epidermal barrier function based on the assessment of single concentrations is not important for the clinical course of AD. Indeed, it is hypothesized that AD cannot be explained by a single mechanism alone; this also suggests that newly developed biological drugs that target a highly specific biological axis of the immune or skin barrier development systems are unlikely to be effective in all patients with $A D$ [37]. However, according to recent reports, maintaining the balance of MMPs/TIMPs leads to the maintenance of the normal epidermal barrier. The MMP-2 defect was observed in this research, which is consistent with the current literature. Therefore, it can be assumed that MMP-2 plays an important role in the course of $A D$, and its concentration regulates the epidermal barrier function.

\section{Conclusions}

Based on the above observations of other authors, and on the outcomes of research presented here, one can draw a cautious conclusion that the severity of AD is not so much correlated with the individual concentrations of MMP-1, MMP-2, TIMP-1, and it is more relevant to assess the balance between MMP and TIMP. The advantage of TIMP-1 over MMP-1 limits TEWL and maintains the correct water content of the epidermis. The correlation between MMP concentration levels and the clinical picture of the disease was demonstrated only for MMP-2, suggesting that MMP-2 plasma concentration may be a marker of the clinical state in patients with atopic dermatitis. The results of this study warrant further investigation. Investigation conducted so far was limited by the inability to identify a serum IgE level in studied patients with atopic dermatitis. A larger patient population will be required to understand the applicability of MMPs measures in routine clinical practice. Although the MMP/TIMP indicator has been associated with the severity of the AD process, it seems necessary to broaden the area of research in order to identify all MMPs and TIMPs, as well as to assess interactions between them. Further research into MMPs inhibitors may underpin their application in a number of dermatological diseases involving damage to the epidermal barrier.

\section{Conflict of interest}

The authors declare no conflict of interest.

\section{References}

1. Sacotte R, Silverberg JI. Epidemiology of adult atopic dermatitis. Clin Dermatol 2018; 36: 595-605.

2. Silverberg JI, Vakharia PP, Chopra R, et al. Phenotypical differences of childhood-and adult-onset atopic dermatitis. J Allergy Clin Immunol Pract 2018; 6: 1306-12.

3. Celakovska J, Bukač J, Ettler K, et al. Atopic dermatitis in adolescents and adults - the evaluation of association with other allergic diseases and parameters. Food Agricult Immunol 2017; 28: 933-48.

4. De Bruin Weller MS, Rockmann H, Knulst AC, et al. Evaluation of the adult patient with atopic dermatitis. Clin Exp Allergy 2013; 43: 279-91.

5. Kobayashi T, Glatz M, Horiuchi K, et al. Dysbiosis and Staphyloccus aureus colonization drives inflammation in atopic dermatitis. Immunity 2015; 42: 756-66.

6. Katoh N, Hirano S, Suehiro M, et al. Increased levels of serum tissue inhibitor of metalloproteinase-1 but not metalloproteinase-3 in atopic dermatitis. Clin Exp Immunol 2002; 127: 283-8.

7. Landriscina A, Rosen J, Albanese J, et al. Identifying new biologic targets in atopic dermatitis (AD): a retrospective histologic analysis. J Am Acad Dermatol 2015; 73: 521-3.

8. Iwai I, Han H, den Hollander L, et al. The human akin barrier is organized as stacked bilayers of fully extended ceramides with cholesterol molecules associated with the ceramide sphingoid moiety. J Invest Dermatol 2012; 132: 2215-25.

9. Rawlings AV, Voegeli R. Stratum corneum proteases and dry skin conditions. Cell Tissue Res 2013; 351: 217-35.

10. Lagente $\mathrm{V}$, Boichot E. Role of matrix metalloproteinases in the inflammatory process of respiratory diseases. J Mol Cell Cardiol 2010; 48: 440-4.

11. Voegeli R, Rawlings A, Breternitz M, et al. Increased stratum corneum serine protease activity in acute eczematous atopic skin. Br J Dermatol 2009; 161: 70-7.

12. Hocking DC, Sottile J, Langenbach KJ. Stimulation of integrin-mediated cell contractility by fibronectin polymerization. J Biol Chem 2000; 275: 10673-82.

13. Rechardt O, Elomaa O, Vaalamo M, et al. Stromelysin-2 is upregulated during normal wound repair and is induced by cytokines. J Invest Dermatol 2000; 115: 778-87.

14. Parks WC. What is the $\alpha 2 \beta 1$ integrin doing in the epidermis? J Investig Dermatol 2007; 127: 264-6.

15. Butler GS, Overall CM. Updated biological roles for matrix metalloproteinases and new "intracellular" substrates revealed by degradomics. Biochemistry 2009; 48: 10830-45.

16. Brunner PM, Suárez-Farińas M, He H, et al. The atopic dermatitis blood signature is characterized by increases in inflammatory and cardiovascular risk proteins. Sci Rep 2017; 7: 8707.

17. Klein T, Bischoff R. Physiology and pathophysiology of matrix metalloproteases. Amino Acids 2011; 41: 271-90. 
18. Collier IE, Legant W, Marmer B, et al. Diffusion of MMPs on the surface of collagen fibrils: the mobile cell surface - collagen substratum interface. PLoS One 2011; 6: e24029.

19. Caterson B, Flannery CR, Hughes CE, et al. Mechanisms involved in cartilage proteoglycan catabolism. Matrix Biol 2000; 19: 333-44.

20. Korpi JT, Kervinen V, Maklin H, et al. Collagenase-2 (matrix metalloproteinase-8) plays a protective role in tongue cancer. Br J Cancer 2008; 98: 766-75.

21. Groblewska M, Mroczko B, Szmitkowski M. The role of selected matrix metalloproteinases and their inhibitors in colorectal cancer development. Postepy Hig Med Dosw 2010; 64: 22-30.

22. Smith WP, Bishop MA, Norton SJ. Cosmetic benefits derived from the topical application of acid proteases. In: Skin Moisturization. $2^{\text {nd }}$ edn. Rawlings AV, Leyden JJ (eds). Informa Healthcare, New York 2009; 397-410.

23. Del Rosso JQ, Levin J. The clinical relevance of maintaining the functional integrity of the stratum corneum in both healthy and disease affected skin. J Clin Aesthet Dermatol 2011; 4: 22-42.

24. Dziankowska-Bartkowiak B, Waszczykowska E, Żebrowska A. The role of metalloproteinases and their inhibitors in the patomechanism of skin diseases. Alergia Astma Immunol 2004; 9: 71-9.

25. Harper JI, Godwin H, Green A, et al. A study of matrix metalloproteinase expression and activity in atopic dermatitis using a novel skin wash sampling assay for functional biomarker analysis. Br J Dermatol 2010; 162: 397-403.

26. Weidinger S, Novak N. Atopic dermatitis. Lancet 2016; 387 : 1109-22.

27. Kim TI, Park HJ, Won YY, et al. Basement membrane status is intact in urticarial dermatitis vs. adult-onset atopic dermatitis. Ann Dermatol 2018; 30: 258-61.

28. Li C, Lasse S, Lee P, et al. Development of atopic dermatitislike skin disease from the chronic loss of epidermal caspase-8. Proc Natl Acad Sci USA 2010; 107: 22249-54.

29. Ratzinger G, Stoitzner P, Ebner S, et al. Matrix metalloproteinases 9 and 2 are necessary for the migration of langerhans cells and dermal dendritic cells from human and murine skin. J Immunol 2002; 168: 4361-71.

30. Tanaka A, Amagai Y, Oida K, et al. Recent findings in mouse models for human atopic dermatitis. Exp Anim 2012; 61: 77-84.

31. Bode W, Fernandez-Catalan C, Grams F, et al. Insights into MMP-TIMP interactions. Ann NY Acad Sci 1999; 878: 73-91.

32. de Oliveira Titz T, Orfali RL, de Lollo C, et al. Impaired CD23 and CD62L expression and tissue inhibitors of metalloproteinases secretion by eosinophils in adults with atopic dermatitis. J Eur Acad Dermatol Venereol 2016; 30: 2072-6.

33. Lan CCE, Fang AH, Wu PH, Wu CS. Tacrolimus abrogates TGF- $\beta 1$-induced type i collagen production in normal human fibroblasts through suppressing p38MAPK signalling pathway: Implications on treatment of chronic atopic dermatitis lesions. J Eur Acad Dermatology Venereol 2014; 28: 204-15.

34. Martins JC, Martins C, Aoki V, et al. Topical tacrolimus for atopic dermatitis. Cochrane Database Syst Rev 2015; 2015 : CD009864.

35. Mempel M, Schmidt T, Boeck K, et al. Changes in collagen I and collagen III metabolism in patients with generalized atopic eczema undergoing medium-dose ultraviolet A1 phototherapy. Br J Dermatol 2000; 142: 473-80.

36. Khattri S, Brunner PM, Garcet S, et al. Efficacy and safety of ustekinumab treatment in adults with moderate-to-severe atopic dermatitis. Exp Dermatol 2017; 26: 28-35.
37. Thijs JL, Strickland I, Bruijnzeel-Koomen CAFM, et al. Moving toward endotypes in atopic dermatitis: identification of patient clusters based on serum biomarker analysis. J Allergy Clin Immunol 2017; 140: 730-7. 